\title{
High Resolution GPU-Based Flow Simulation of the Gaseous Methane-Oxygen Detonation Structure
}

Keywords Detonation · Cellular Structure · Instability · Computational Fluid Dynamics

\section{Introduction}

A gaseous detonation is a combustion-driven, compressible wave propagating at supersonic speeds. Experimental visualizations and numerical simulations confirm that detonation waves have a complex, multi-dimensional, cellular structure generated by various gasdynamic interactions (Ohyagi et al. 2000; Ng \& Zhang 2012). Except in special combustible mixtures (such as argon-diluted mixtures) with a "laminar" detonation front and regular cellular pattern, the fine-scale cellular structure of unstable detonations remains difficult to describe due to its turbulent nature with large hydrodynamic fluctuations and unreacted pockets (Gamezo et al. 1999; Radulescu et al. 2005). Fundamentally, it is desirable to determine a characteristic length scale for the unstable cellular detonation so as to correlate with other detonation dynamic parameters such as direct initiation energy or detonability limits (Lee \& Radulescu 2005).

Numerical simulations of cellular detonations have been performed since the 80's, see Ng \& Zhang (2012) and references therein. The detonation model is generally the reactive Euler equations with one-step chemical kinetics. This model has proven useful in capturing the salient features of cellular detonations: the triple-wave configuration at the leading front, and the unburned pockets within the detonation structure. However, despite advanced numerical techniques like adaptive mesh refinement (AMR), previous simulations suffer from a lack of numerical resolution to properly reveal the hydrodynamic instabilities and pressure wave interactions within or behind the irregular detonation structure. These instabilities have recently been found to be essential in the propagation mechanism of detonations (Radulescu et al. 2005; Kiyanda \& Higgins 2013) and a large number of computation cells at the detonation front are inevitably required to resolve the structure (Mahmoudi \& Mazaheri 2011; Kessler et al. 2011; Mazaheri et al. 2012).

In this paper, high-resolution numerical simulations are reported to elucidate the dynamics of unstable gaseous methane-oxygen detonations. Comparisons of the numerical detonation structure with experimental photography are presented. The computation is performed using Graphic Processing Units (GPU). The computational Cartesian geometry used makes the many GPU cores convenient to perform computations leading to significant speed-ups over a conventional CPU (Vanka 2013). GPU computing allows the use of a high-resolution mesh throughout the complete domain. It eliminates the use of AMR and hence, any possible adaptive grid-induced, numerical artifacts.

\section{Methods}

As for previous studies (see, Ng \& Zhang 2012), numerical simulations were performed using the reactive Euler equations with a one-step Arrhenius reaction model. The grid size used in this study is $62.5 \mu$ m, yielding a resolution of 148 points per ZND half reaction zone length $L_{1 / 2}$ where half of the reactant is consumed. A sufficiently long domain length of $x=0.8 \mathrm{~m}$ (about $86 L_{1 / 2}$ ) moving at the CJ detonation velocity is used to avoid any disturbance to the front from the back boundary. A region of overpressure $2 P_{\mathrm{CJ}}$ with an embedded frontal perturbation of sinusoidal waveform (e.g., amplitude $a=3 \mathrm{~mm}$ and wavelength $\Omega=25 \mathrm{~mm}$ ), to facilitate detonation formation, covers three quarters of the domain length from the left edge. Sufficient computational time was used to allow detonation propagation for more than $400 L_{1 / 2}$. No explicit molecular diffusion or turbulence model is included and the model is

C.B. Kiyanda $\cdot$ H.D. $\mathrm{Ng}^{\dagger}$

Department of Mechanical and Industrial Engineering, Concordia University, Montréal, QC, H3G 1M8 Canada

†e-mail: hoing@encs.concordia.ca

G.H. Morgan · N. Nikiforakis

Cavendish Laboratory, Department of Physics, University of Cambridge, Cambridge, UK 
restricted only to compressible gas dynamic and reactive phenomena (Radulescu \& Maxwell 2011). The initial condition and the 10-cm width of the computational domain mirror the experiment. The top and bottom boundaries are reflective, while a constant inflow at speed $D_{\mathrm{CJ}}$ is defined at the right boundary. The left boundary is relaxed toward the inflow conditions following Gamezo et al. (1999). The established detonation wave thus remains at approximately the same location in the domain. The chemical parameters are obtained by calibrating the one-step model against the Konnov chemical kinetic mechanism (Konnov 1998), following Radulescu \& Maxwell (2011). These are: $\gamma=1.17$, heat release $Q / R T_{0}=59.6$ and activation energy $E_{\mathrm{a}} / R T_{0}=49.5$ for methane-oxygen mixtures at $P_{\mathrm{o}}$ $=3.4 \mathrm{kPa}$ and $T_{\mathrm{o}}=300 \mathrm{~K}$. The governing equations are discretized on a Cartesian uniform grid and solved with the MUSCL-Hancock scheme with first-order dimensional splitting. A hybrid HLLC-HLLE solver is used for the Riemann problem (Toro 2009). A CFL number of 0.80 is used. The numerical code is implemented using the CUDA programming language (NVIDIA Corp. 2014) and is structured to run on a NVIDIA Tesla C2075 GPU. This device has 14 streaming multiprocessors or equivalently 448 CUDA cores with 6GB global memory. For the computation with the present algorithm, the solution for each cell is performed by a single thread, with threads logically grouped into blocks of 64 threads capable of inter-thread communication. By executing multiple blocks at the same time, the GPU is able to "swap" between blocks and hide memory latency. With compute capability 2.0, 512 resident threads can be executed in parallel per streaming multiprocessor on this GPU. The simulation took about 1 day of runtime.

Experimental photographs from an earlier study (experimental details in Kiyanda \& Higgins 2013) were compared to the numerical results. The experimental images were obtained using a rectangular cross-sectional channel of $100 \mathrm{~mm}$ by $25 \mathrm{~mm}$. The transparent channel walls permitted direct visualization of the propagating detonation. The mixture was stoichiometric methane-oxygen representative of highly unstable detonations with an irregular cell pattern (Radulescu et al. 2005). It was prepared beforehand in a mixing tank via the partial pressure method. The initial state for the experiment is also $P_{\mathrm{o}}=3.4 \mathrm{kPa}$ and $T_{\mathrm{o}}=300 \mathrm{~K}$. A detonation was initiated by a high energy spark discharge. Schlieren photography with a 320-mm diameter field of view was used to visualize the detonation structure. A high-speed digital framing camera (HSFC Pro) was used to obtain eight consecutive Schlieren images. A high-speed digital framing camera (DICAM Pro) was also set to record the light emitted from the regions of high chemical activity inside the detonation structure.

\section{Results and discussion}

Figure 1 shows the comparison between experimentally obtained flow structures and numerical simulations. A sequence of Schlieren frames shows the evolution of the unstable dynamic structure. As first seen in the numerical temperature maps, the flow field behind the detonation front involves vortices of different scales generated from the Mach and other shock reflections, Kelvin-Helmholtz instabilities along the shear layers, and Richtmyer-Meshkov instabilities by different gasdynamic interactions. Using GPU-accelerated simulations, these small-scale vortices in the detonation product flow were resolved in this study throughout the domain at high resolution. This allowed the current simulation to capture the transverse pressure wave fluctuations and mixing activity taking place far downstream of the leading shock, where "far downstream" can be several times the distance between the leading front and the furthest cold gas region, as seen in the numerical Schlieren images of Fig. 1. Those cold regions near the front are the unreacted gas pockets cut off by the interaction of transverse waves from the previous cell cycle. These pockets generate tongue-like shapes and burn downstream, as can be clearly seen in both the numerical simulation and experimental observations. These agree well with results reported in the literature (Radulescu et al. 2005; Kiyanda \& Higgins 2013; Kessler et al. 2011). Figure 2, showing also the density contour superimposed with the unburned reactant fraction (simulation) and the self-emitted light (experiment), better reveals the formation of these tongue-like pockets along the primary shear layer inside the detonation structure. Given the high activation energy, the energy release occurs at the edge of the unreacted region shown in white in Fig. 2a (bottom), in accord with the selfluminescent image in Fig. 2b (bottom). Overall, high-resolution, numerical simulations of the irregular detonation structure with GPU reproduce well the results from experimental visualizations. Although no diffusion term or turbulent model are considered in the simulation, the unburned pockets can still be burned through the interaction with resolved, compressible turbulence further downstream (e.g., shock-shock, shock-vortex interaction), effects often under-estimated by the use of AMR in the detonation product flow.

It has been suggested in previous studies that the combustion mechanism of detonations with highly irregular cellular structures, such as is the case for this methane-oxygen mixture, requires the action of turbulent diffusion (Radulescu et al. 2005; Mazaheri et al. 2012). Some authors have thus resorted to simulating such detonation structures using the Navier-Stokes equations and an artificially high diffusion term (Mazaheri et al. 2012). The reduction of the diffusion associated with the numerical discretization has been offered as an explanation for the inability to propagate a highly unstable detonation in simulations using a locally high resolution (Radulescu et al. 2005). This resolution limit, above which detonations could not propagate, was at roughly 64 points per $L_{1 / 2}$. 


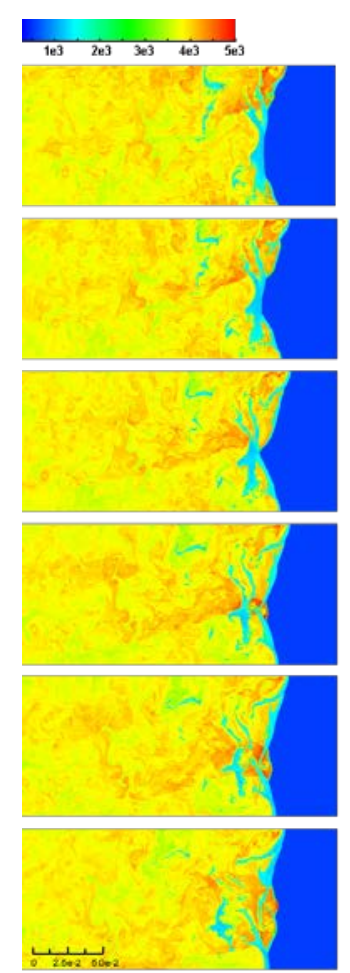

(a)

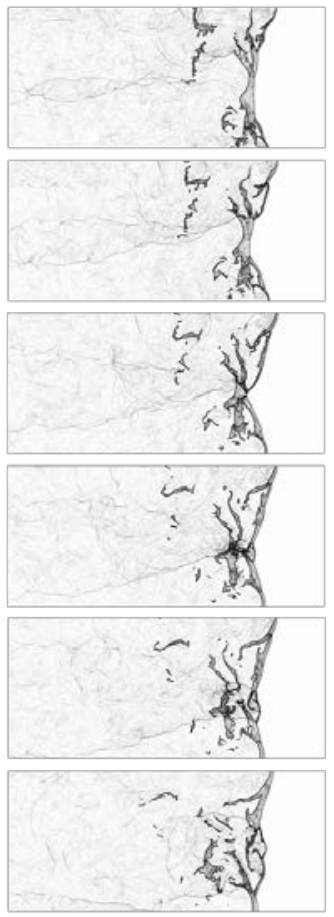

(b)

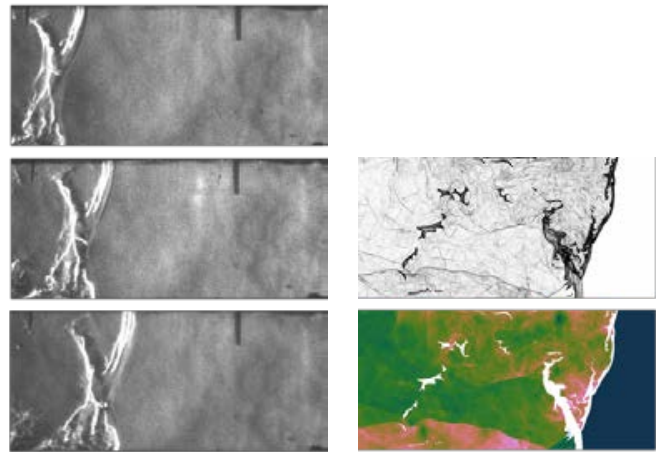

(a)

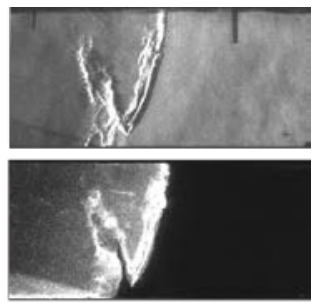

(b)

FIGURE 2. a) Schlieren plot and density contour combined with the reactant fraction from numerical simulations and b) Schlieren and self-luminescent photographs from experiments.

FIGURE 1. A sequence of detailed structure of the unstable methane-oxygen detonation. a) and b) temperature and Schlieren plots from numerical simulations; and c) experimental Schlieren photographs.

A resolution study shows that, for a low grid resolution of 16 points per $L_{1 / 2}$, no large, reactive pockets are present in the flow field. All material is burned due to numerical diffusion. By increasing the grid resolution, hydrodynamic instabilities with vortices of different scales behind the detonation front are becoming more apparent together with the appearance of unburned pockets. As the resolution is increased, the numerical diffusion diminishes. In turn, ignition requires the presence of instabilities of sufficient strength to fragment and burn these pockets. When properly numerically resolved, the role of small-scale vortices and the interactions of pressure waves with the density interfaces associated with unreacted pockets are sufficient to increase the level of turbulence in the flow and promote combustion. In this case, detonation propagation is achieved with a uniform grid resolution about 2.5 times denser as that of Radulescu et al. (2005). The turbulence activity is thus not only an important mechanism near the front, i.e., at the triple point and shear layers, but throughout the front. Turbulent flow downstream of the front appears to be significant as well and needs to be resolved in order to consume the unreacted pockets and sustain the propagation of highly unstable detonations. This conclusion is in agreement with those previously cited studies when it comes to the nature of the mode of combustion. We caution, though, that care must be exercised when simulating such detonation structures. At least for the purposes of AMR, what is considered "close to the leading front" must be carefully examined and numerically resolved. Lastly, it is worth noting that the present use of GPU computing accelerates the high-resolution simulation of unstable cellular detonation, performed with relatively short runtime. However, the available GPU global memory still limits the size (or even higher resolution) of the problem we can simulate. The future work is therefore to develop memory-efficient parallel programming on distributed multi-GPUs.

Acknowledgment This work is supported by NSERC (Canada) and NVIDIA academic hardware donation program.

\section{References}

Gamezo VN, Desbordes D and Oran ES (1999) Formation and evolution of two-dimensional cellular detonations. Combust Flame 116:154-165.

Kessler DA, Gamezo VN and Oran ES (2011) Multilevel detonation cell structures in methane-air mixtures. Proc Comb Inst 33:2211-2218.

Kiyanda CB and Higgins AJ (2013) Photographic investigation into the mechanism of combustion in irregular detonation waves. Shock Waves 23:115-130. 
Konnov AA (1998) Detailed reaction mechanism for small hydrocarbons combustion. Release 0.4, 1998, http://homepages.vub.ac.be/ akonnov/

Lee JHS and Radulescu MI (2005) On the hydrodynamic thickness of cellular detonations. Combust Expl Shock Wave 41:745-765.

Mahmoudi Y and Mazaheri K (2011) High resolution numerical simulation of the structure of 2-D gaseous detonations. Proc Comb Inst 33:2187-2194.

Mazaheri K, Mahmoudi Y and Radulescu MI (2012) Diffusion and hydrodynamic instabilities in gaseous detonations. Combust Flame 159:2138-2154.

Ng HD and Zhang F (2012) Detonation Instability. In: Shock Wave Science and Technology Reference Library, Vol. 6. Edited by Zhang F, Berlin-Heidelberg, Springer, pp. 107-212.

Ohyagi S, Kobayashi Y and Obara T (2000) Numerical visualization of decaying process of oxyhydrogen detonation waves. J Vis 3(2):100.

Radulescu MI, Sharpe GJ, Lee JHS, Kiyanda CB, Higgins AJ and Hanson RK (2005) The ignition mechanism in irregular structure gaseous detonations. Proc Comb Inst 30:1859-1867.

Radulescu MI and Maxwell BM (2011) The mechanism of detonation attenuation by a porous medium and its subsequent re-initiation. J Fluid Mech 667:96-134.

Toro EF (2009) Riemann Solvers and Numerical Methods for Fluid Dynamics. $3^{\text {rd }}$ edition, Springer-Verlag, Berlin. Vanka SP (2013) Computational fluid dynamics on graphics processing units. J Fluids Eng 135, 061401.

NVIDIA Corporation (2014) NVIDIA CUDA Programming Guide, http://developer.nvidia.com/cuda 